\title{
TAUBERIAN CONCLUSIONS
}

\author{
K. A. JUKES AND I. J. MADDOX
}

F or Professor B. Kuttner on his retirement

ABSTRAC.T. Littlewood's celebrated Tauberian theorem states that $\Sigma a_{n}=s$ (Abel) and $n a_{n}=O(1)$ imply $s_{n}=\Sigma_{k=1}^{n} a_{k}$ converges to $s$, the Tauberian condition $n a_{n}=O(1)$ being best possible. We investigate 'best possibility' of the conclusion $s_{n}-s=o(1)$, replacing the usual Tauberian condition by $\left(q_{n}{ }_{n}\right) \in E$ where $\left(q_{n}\right)$ is a positive sequence and $E$ a given sequence space.

1. Introduction. The first Tauberian theorem of $O$ type was proved by Hardy [1]. It asserts that $\Sigma a_{n}=s(C, k)$ and $n a_{n}=O(1)$ imply $s_{n}=\sum_{k=1}^{n} a_{k}$ converges to $s$. Hardy raised the question as to whether $(C, k)$ summability could be replaced by Abel summability, stating he was inclined to think it could not. However, Littlewood, in his now famous paper [3], showed that $\Sigma a_{n}=s$ (Abel) and $n a_{n}=O(1)$ implied $s_{n} \rightarrow s$, and also, that the big $O$ condition was best possible in that if $0<\phi(n) \rightarrow \infty$, there exists a divergent $\sum a_{n}$ such that $n\left|a_{n}\right|<\phi(n)$ and $\Sigma a_{n}$ is Abel-summable.

In the present note we consider the 'best possibility' of certain Tauberian conclusions. One of the simplest questions is to ask whether $\Sigma a_{n}=0$ (Abel) and $n a_{n}=O(1)$ imply that $s_{n}=o(1)$ is best possible. That this is so is a special case of Corollary 1 below.

Let $q=\left(q_{n}\right)$ and $p=\left(p_{n}\right)$ denote sequences of positive real numbers. Writing $a=\left(a_{n}\right)$ and $q a=\left(q_{n} a_{n}\right)$, for a given sequence space $E$ let $S(q, E)=\left\{a: q a \in E\right.$ and $\left.s_{n} \rightarrow 0\right\}$. Denote by $P(q, E)$ the property that for all $p \notin l_{\infty}, \exists a \in S(q, E)$ such that $p_{n} s_{n} \nrightarrow 0$. Our main aim is to establish results of the form: $P(q, E)$ if and only if $1 / q=\left(1 / q_{n}\right) \notin E^{\prime}$, where $E^{\prime}$ is another sequence space which is, in a sense, dual to $E$.

In the following table we list corresponding spaces $E, E^{\prime}$. Here $\gamma=$ $\left\{a: \sum a_{n}\right.$ converges $\}$ and $B V_{0}=\left\{a: \Sigma\left|\Delta a_{n}\right|<\infty\right.$ and $\left.a_{n} \rightarrow 0\right\}$ where $\Delta a_{n}=a_{n}-a_{n+1}$.

2. Verification of the table. In proving the results in the table we shall use the negation of $P(q, E)$, which we denote by $Q(q, E)$, namely $\exists p \notin l_{\infty}$ such that for all $a \in S(q, E), p_{n} s_{n} \rightarrow 0$.

(i) To prove $P\left(q, l_{r}\right)$ implies $1 / q \notin c_{0}(0<r \leq 1)$, we show equivalently

Received by the editors October 9, 1974.

AMS (MOS) subject classifications (1970). Primary 40E05; Secondary 40G10, 40G99.

Key words and phrases. Tauberian theorems, best possible conclusions, Abel, Ingham methods. 


\begin{tabular}{|c|c|}
\hline$E$ & $E^{\prime}$ \\
\hline$l_{r}(0<r \leq 1)$ & $c_{0}$ \\
\hline$l_{r}(1<r<\infty)$ & $l_{s}(1 / r+1 / s=1)$ \\
\hline$l_{\infty}$ & $l_{1}$ \\
\hline$c_{0}$ & $l_{1}$ \\
\hline$\gamma$ & $B V_{0}$ \\
\hline
\end{tabular}

that $1 / q \in c_{0}$ implies $Q\left(q, l_{r}\right)$. If $1 / q \in c_{0}$, we may choose $p_{n}$ so that $1 / p_{n}=\sup _{k \geq n+1} 1 / q_{k}$. For then, if $a \in S\left(q, l_{r}\right)$, given $\epsilon>0$,

$$
\left|s_{n}\right|^{r}=\left|\sum_{n+1}^{\infty} a_{k}\right|^{r} \leq\left(\frac{1}{p_{n}}\right)^{r} \sum_{n+1}^{\infty}\left|q_{k} a_{k}\right|^{r}<\epsilon\left(\frac{1}{p_{n}}\right)^{r} \quad\left(n>n_{0}, \text { say }\right),
$$

on using $b, c \geq 0$ implies $(b+c)^{r} \leq b^{r}+c^{r}$ (see e.g. Maddox [4, p.22]).

Conversely, suppose $1 / q \notin c_{0}$ and $p \notin l_{\infty}$. Then there exists $c>0$ and $\left(m_{i}\right)$ strictly increasing such that $q_{m_{i}} \equiv q\left(m_{i}\right) \leq c$ (each $\left.i\right)$. Choose $k_{1}>1$ such that $p\left(k_{1}\right)>2$ and then $m_{i}>k_{1}$ such that $q\left(m_{i}\right) \leq c$. Denote $m_{i}$ by $t_{1}$. Let

$$
a_{k}= \begin{cases}0 & \left(1 \leq k<t_{1}\right) \\ 1 / p\left(k_{1}\right) & \left(k=t_{1}\right)\end{cases}
$$

For $n>1$ choose $k_{n}>t_{n-1}$ such that $p\left(k_{n}\right)>2 p\left(k_{n-1}\right)$ and then $m_{j}>k_{n}$ such that $q\left(m_{j}\right) \leq c$. Denote $m_{j}$ by $t_{n}$. Let

$$
a_{k}= \begin{cases}0 & \left(t_{n-1}<k<t_{n}\right), \\ 1 / p\left(k_{n}\right)-1 / p\left(k_{n-1}\right) & \left(k=t_{n}\right) .\end{cases}
$$

Then $s_{n}$ decreases $\left(n>t_{1}\right), s\left(t_{n}\right)=1 / p\left(k_{n}\right)<1 / 2^{n}$ and for $n>1, s\left(k_{n}\right)=$ $1 / p\left(k_{n-1}\right)>2 / p\left(k_{n}\right)$. Further for $n \geq 2$,

$$
\left|q\left(t_{n}\right)\left(1 / p\left(k_{n}\right)-1 / p\left(k_{n-1}\right)\right)\right| \leq c / p\left(k_{n-1}\right) \leq c / 2^{n-1},
$$

whence $q a \in l_{r}$, and so $P\left(q, l_{r}\right)$ holds.

(ii) Suppose $a \in S\left(q, l_{r}\right)(1<r<\infty)$ and $1 / q \in l_{s}(1 / r+1 / s=1)$.

Taking $p_{n}=\left(\sum_{n+1}^{\infty} 1 / q_{k}^{s}\right)^{-1 / s}, Q\left(q, l_{r}\right)$ holds. For by Hölder's inequality, given $\epsilon>0$,

$$
\left|s_{n}\right|=\left|\sum_{n+1}^{\infty} a_{k}\right| \leq\left(\sum_{n+1}^{\infty}\left|q_{k} a_{k}\right|^{r}\right)^{1 / r} / p_{n}<\frac{\epsilon}{p_{n}} \quad\left(n>n_{0}, \text { say } .\right.
$$

Conversely, let $1 / q \notin l_{s}$ and $p \notin l_{\infty}$. Write 


$$
M(m, n)=\sum_{m+1}^{n} \frac{1}{q_{k}^{s}} \quad(n \geq m+1) .
$$

Put $a_{1}=0$. Choose $n_{1}>1$ so that $Y\left(1, n_{1}\right)>1$ and $p\left(n_{1}\right)>1$. Put

$$
a_{k}=1 / M\left(1, n_{1}\right) p\left(n_{1}\right) q_{k}^{s} \quad\left(2 \leq k \leq n_{1}\right) .
$$

For $i \geq 1$ choose $n_{i+1}>n_{i}$ so that $M\left(n_{i}, n_{i+1}\right)>1$ and $p\left(n_{i+1}\right)>2 p\left(n_{i}\right)$. Put

$$
a_{k}=\frac{\left(1 / p\left(n_{i+1}\right)-1 / p\left(n_{i}\right)\right)}{M\left(n_{i}, n_{i+1}\right) q_{k}^{s}} \quad\left(n_{i}<k \leq n_{i+1}\right) .
$$

Then $s_{n}$ decreases $\left(n>n_{1}\right)$ and $s\left(n_{i+1}\right)=1 / p\left(n_{i+1}\right)$. Further

$$
\begin{aligned}
\sum_{n_{i}+1}^{n_{i+1}}\left|q_{k} a_{k}\right|^{r} & \leq \sum_{n_{i}+1}^{n_{i+1}} 1 / M^{r}\left(n_{i}, n_{i+1}\right) q_{k}^{r(s-1)} p^{r}\left(n_{i}\right) \\
& \left.=1 / \mathrm{M}^{r-1}\left(n_{i}, n_{i+1}\right) p^{r(} n_{i}\right)<1 / p^{r}\left(n_{i}\right)<1 / 2^{r(i-1)},
\end{aligned}
$$

whence $q a \in l_{r}$.

(iii) Suppose $a \in S\left(q, l_{\infty}\right)$ and $1 / q \in l_{1}$. Taking $p_{n}=\left(\sum_{n+1}^{\infty} 1 / q_{k}\right)^{-1 / 2}$, we have $p_{n} \rightarrow \infty$ and $\left|p_{n} s_{n}\right| \leq\left(\sup _{n}\left|q_{n} a_{n}\right|\right) p_{n}^{-1 / 2} \rightarrow 0$, and so $Q\left(q, l_{\infty}\right)$ holds.

Conversely, let $1 / q \notin l_{1}$ and $p \notin l_{\infty}$. Let $a$ be as constructed in (ii) above, taking $s=1$. Then again $s_{n}$ decreases $\left(n>n_{1}\right)$ and $s\left(n_{i+1}\right)=$ $1 / p\left(n_{i+1}\right)$. Further for $n_{i}<k \leq n_{i+1},\left|q_{k} a_{k}\right| \leq 1 / p\left(n_{i}\right) \rightarrow 0$. Hence $P\left(q, l_{\infty}\right)$ holds.

(iv) Suppose $1 / q \in l_{1}$. Then $Q\left(q, c_{0}\right)$ trivially holds with $1 / p_{n}=$ $\sum_{n+1}^{\infty} 1 / q_{k}$. Conversely, if $1 / q \notin l_{1}$, then $P\left(q, c_{0}\right)$ holds with $a$ as in (iii).

(v) Suppose $a \in S(q, \gamma)$ and $1 / q \in B V_{0}$. Write $Q_{n}=\Sigma_{n+1}^{\infty}\left|\Delta\left(1 / q_{k}\right)\right|$. For $m \geq n+2$,

$$
\sum_{k=n+1}^{m} a_{k}=\frac{1}{q_{m}} \sum_{k=n+1}^{m} q_{k} a_{k}+\sum_{k=n+1}^{m-1}\left(\sum_{r=n+1}^{k} q_{r} a_{r}\right) \Delta\left(\frac{1}{q_{k}}\right) .
$$

So given $\epsilon>0$, for $n>n_{0}(\epsilon)$,

$$
\left|\sum_{k=n+1}^{m} a_{k}\right|<\epsilon\left(\frac{1}{q_{m}}+\sum_{n+1}^{m-1}\left|\wedge\left(\frac{1}{q_{k}}\right)\right|\right)
$$

whence

$$
\left|s_{n}\right|=\left|\sum_{n+1}^{\infty} a_{k}\right| \leq \epsilon Q_{n} .
$$

Note that $Q_{n}=0$ implies $q_{k}=q_{n+1}(k>n)$, contradicting $1 / q \in c_{0}$. So $Q(q, y)$ holds with $p_{n}=1 / Q_{n}$.

Conversely suppose $1 / q \notin B V_{0}$ and $p \notin l_{\infty}$. Either

(a) $\Sigma\left|\Delta\left(1 ; q_{k}\right)\right|<\infty$ and $1 / q_{k} \nrightarrow 0$, or

(b) $\Sigma\left|\Delta\left(1 / q_{k}\right)\right|=\infty$. 
Now $\Sigma\left|\Delta\left(1 / q_{k}\right)\right|<\infty$ implies $1 / q \in c$ and so (a) implies $q \in l_{\infty}$. Further, (a) implies $1 / q \notin l_{1}$. But the sequence $a$ constructed in (iii) satisfies $a \in l_{1}$. Since $q \in l_{\infty}$, then $q a \in l_{1}$. In particular (a) implies $P(q, \gamma)$.

When (b) holds, first suppose $1 / q \notin c_{0}$. Then $P\left(q, l_{r}\right)(0<r \leq 1)$ holds But $q a \in l_{r}$ implies $q a \in \gamma$ and so $P(q, \gamma)$ holds. Now suppose $1 / q \in c_{0}$. Writing $t_{n}=\sum_{k=1}^{n} q_{k} a_{k}$, we have

$$
s_{n}=\sum_{1}^{n} a_{k}=\frac{{ }^{t} n}{q_{n}}+\sum_{k=1}^{n-1} t_{k} \Delta\left(\frac{1}{q_{k}}\right) \quad\left(n \geq 2^{*}\right) .
$$

Define $\operatorname{sgn} z=|z| / z(z \neq 0)$, sgn $0=0$. Choose $n_{1}>2$ such that $p\left(n_{1}\right)>1$ and $\Sigma_{1}^{n_{1}-1}\left|\Lambda\left(1 / q_{k}\right)\right|>1$. Define

$$
t_{k}=\left\{\begin{array}{l}
\left(\operatorname{sgn} \Delta\left(1 / q_{k}\right)\right) p\left(n_{1}\right) \Sigma_{1}^{n_{1}-1}\left|\Delta\left(1 / q_{k}\right)\right| \quad\left(1 \leq k<n_{1}\right), \\
0 \quad\left(k=n_{1}\right) .
\end{array}\right.
$$

For $r \geq 1$, choose $n_{r+1}>n_{r}$ such that $\sum_{n_{r}+1}^{n_{r+1}-1}\left|\Delta\left(1 / q_{k}\right)\right|>1$ and $p\left(n_{r+1}\right)>$ $2 p\left(n_{r}\right)$. Define

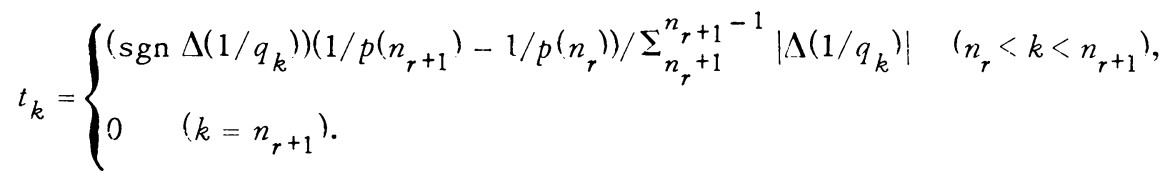

Then $s\left(n_{r+1}\right)=1 / p\left(n_{r+1}\right), t_{n} \rightarrow 0, t_{n} / q_{n} \rightarrow 0$ and $\sum_{1}^{n-1} t_{k} \Delta\left(1 / q_{k}\right) \rightarrow 0$. Hence $P(q, \gamma)$ holds, completing the table.

For corresponding spaces $E, E^{\prime}$ we immediately have

Corollary 1. Suppose $\Sigma a_{n}=0(A)$ and qa $\in$ E imply $s_{n}=o(1)$, where $A$ is a regular summability method. If $1 / q \notin E^{\prime}$, then the conclusion $s_{n}=$ $o(1)$ is best possible.

If we take $A$ to be the Abel method, $q=(n)$ and $E=l_{\infty}$ in Corollary 1, then we have the answer to our question in the introduction regarding the best possible nature of $s_{n}=o(1)$ in the classical Tauberian case.

A later Tauberian theorem of Ingham's [2, Theorem 2] states that $\Sigma a_{n}=s(I)$ and $n a_{n}=O(1)$ imply $s_{n} \rightarrow s$, where (I) denotes Ingham summability. Taking $a_{n}=\mu(n) / n$, where $\mu(n)$ is the Möbius function, gives the prime number theorem. Although $(I)$ is not regular, for $E=l_{r}(0<r \leq \infty)$ or $c_{0}$, we do have

Corollary 2. Suppose $\Sigma a_{n}=0(I)$ and $q a \in E$ imply $s_{n}=o(1)$. If $1 / q \notin E^{\prime}$, then the conclusion $s_{n}=o(1)$ is best possible.

The proof follows immediately, using the following lemma, since in (i) - (iv) above each constructed $\left(a_{k}\right) \in l_{1}$. 
Lemma 1. $\Sigma\left|a_{k}\right|<\infty$ implies $\Sigma a_{k}$ is summable (I).

Proof.

$$
I_{n} \equiv \frac{1}{n} \sum_{k=1}^{n} k a_{k}\left[\frac{n}{k}\right]=\sum_{k=1}^{n} a_{k}+O\left(\frac{1}{n} \sum_{k=1}^{n} k\left|a_{k}\right|\right) .
$$

But $\Sigma\left|a_{k}\right|<\infty$ implies $k\left|a_{k}\right| \rightarrow 0(C, 1)$. Hence $I_{n}:=s_{n}+o(1)$.

3. Best possibility when $1 / q \in E^{\prime}$. If $1 / q \in E^{\prime}$, then $a \in S(q, E)$ does not imply that $s_{n}=o(1)$ is best possible. It is interesting to consider what is best possible in this case. As an illustration we have, when $E=c_{0}$, the following

Theorem. Suppose $1 / q \in l_{1}$. Write $R(n)=\Sigma_{n+1}^{\infty} 1 / q_{k}$. Then:

(a) $a \in S\left(q, c_{0}\right)$ implies $s_{n}=o(R(n))$.

(b) For all $p \notin l_{\infty}, \exists a \in S\left(q, c_{0}\right)$ such that $p_{n^{\prime}}{ }_{n^{\prime}} / R(n) \nrightarrow 0$.

Proof. (a) was noted in (iv) above.

(b) $\mathbb{W}_{\text {rite }} M(m, n)=\sum_{m+1}^{n} 1 / q_{k}(n \geq m+1)$. Choose $n_{1}>1$ so that $p\left(n_{1}\right)>1$. Define $a_{k}=R\left(n_{1}\right) / M\left(0, n_{1}\right) p\left(n_{1}\right) q_{k}\left(1 \leq k \leq n_{1}\right)$. For $r \geq 1$ choose $n_{r+1}>n_{r}$ so that $R\left(n_{r}\right) / M\left(n_{r}, n_{r+1}\right)<2$ and $p\left(n_{r+1}\right)>2 p\left(n_{r}\right)$. Define

$$
a_{k}=\frac{\left(R\left(n_{r+1}\right) / p\left(n_{r+1}\right)-R\left(n_{r}\right) / p\left(n_{r}\right)\right)}{M\left(n_{r}, n_{r+1}\right) q_{k}} \cdot \quad\left(n_{r}<k \leq n_{r+1}\right) .
$$

Then, for $n_{r}<k \leq n_{r+1},\left|q_{k} a_{k}\right| \leq 2 / p\left(n_{r}\right) \rightarrow 0$, while $s_{n}$ decreases $\left(n>n_{1}\right)$ and $s\left(n_{r}\right)=R\left(n_{r}\right) / p\left(n_{r}\right)$, completing the proof.

Finally, we have

Corollary 3. Suppose $1 / q \in l_{1}$.

(a) If $q a \in c_{0}$ and $\Sigma a_{n}=0(I)$, then $s_{n}=o(R(n))$.

(b) For all $p \notin l_{\infty}, \exists a$ such that $q a \in c_{0}, \Sigma a_{n}=0(I)$ but $p_{n_{n}}{ }^{\prime} R(n) \nrightarrow 0$.

This corollary is an immediate consequence of the above Theorem since one can easily establish

Lemma 2. If $1 / q \in l_{1}$, then $q a \in c_{0}$ implies $s_{n}-I_{n} \rightarrow 0$.

\section{REFERENCES}

1. G. H. Hardy, Theorems relating to the summability and convergence of slowly oscillating series, Proc. London Math. Soc (2) 8 (1910), 301-320.

2. A. E. Ingham, Some Tauberian theorems connected with the prime number theorem, J. London Math. Soc. 20 (1945), 171-18n. MR 8, 147.

3. J. E. Littlewood, The converse of Abel's theorem on pou'er series, Proc. London Math. Soc. (2) $10(1910 / 11), 434-448$.

4. I. J. Maddox, Elements of functional analysis, Cambridge, 1970.

DEPARTMENT OF PURE MATHEMATICS, QUEEN'S UNIVERSITY, BELFAST, UNITED KINGDOiM (Current address of I. J. Maddox)

Current address (Dr. K. A. Jukes): Department of Mathematics and Statistics, Sheffield Polytechnic, Sheffield, England 